\title{
Platelet count, aspirin use, and characteristics of host inflammatory responses in colorectal cancer
}

\author{
Juha P. Väyrynen ${ }^{1,2,3^{*}}$ (0, Sara A. Väyrynen ${ }^{1,2,4}$, Päivi Sirniö ${ }^{1,2}$, Ilkka Minkkinen ${ }^{1,2}$, Kai Klintrup ${ }^{5,6}$, Toni Karhu, \\ Jyrki Mäkelä5,6, Karl-Heinz Herzig7,8,9, Tuomo J. Karttunen 1,2, Anne Tuomisto ${ }^{1,2}$ and Markus J. Mäkinen 1,2
}

\begin{abstract}
Background: Platelets not only contribute to hemostasis but also to the regulation of inflammatory reactions and cancer pathogenesis. We hypothesized that blood platelet count would be associated with systemic inflammation, the densities of tumor infiltrating immune cells, and survival in colorectal cancer (CRC), and these relationships could be altered by aspirin use.

Methods: We measured blood platelet count in a cohort of 356 CRC patients and analyzed its relationships with tumor and patient characteristics including aspirin use, markers of systemic inflammation (modified Glasgow Prognostic Score, mGPS; serum levels of CRP, albumin, and 13 cytokines), blood hemoglobin levels, five types of tumor infiltrating immune cells (CD3, CD8, FoxP3, Neutrophil elastase, mast cell tryptase), and survival.

Results: Platelet count inversely correlated with blood hemoglobin levels $(p<0.001)$ and positively correlated with serum levels of CRP and multiple cytokines including IL-1RA, IL-4, IL-6, IL-7, IL-8, IL-12, IFNy, and PDGF-BB ( $p<0.001$ for all), while aspirin use was not associated with the levels of systemic inflammatory markers. High platelet count was also associated with high mGPS $(p<0.001)$ but did not show statistically significant multivariable adjusted associations with the densities of tumor infiltrating immune cells. Higher platelet counts were observed in higher tumor stage ( $p<0.001)$, but platelet count or aspirin use were not associated with patient survival.
\end{abstract}

Conclusions: High platelet count is associated with systemic inflammation in CRC. This study could not demonstrate statistically significant associations between platelet count, aspirin use, and the densities of tumor infiltrating immune cells.

Keywords: Thrombocytosis, Colorectal cancer, Inflammation, Prognosis, Aspirin, Platelet, CRP, Cytokine

\section{Background}

Colorectal cancer (CRC) is one of the most common malignancies and causes of cancer death [1]. The most important prognostic factor is tumor stage $[2,3]$. However, each tumor and patient is unique [4], and more exact categorization based on the features of the tumor and the host could improve our understanding on CRC

\footnotetext{
*Correspondence: juha.vayrynen@oulu.fi

${ }^{1}$ Cancer and Translational Medicine Research Unit, University of Oulu, POB 5000, 90014 Oulu, Finland

Full list of author information is available at the end of the article
}

and enable more individualized disease classifications and treatments.

Platelets are anucleate cell fragments generated by megakaryocytes in the bone marrow and also in other organs including the lung $[5,6]$. Their primary function is to contribute to hemostasis and prevent bleeding $[5,7]$. However, they are also involved in multiple other physiological processes, including inflammation, immunity, angiogenesis, and vessel remodeling [7]. Thrombocytosis, i.e., increased blood platelet count, is either reactive (secondary thrombocytosis) or caused by a clonal bone marrow (myeloproliferative) disorder [8]. The drivers of secondary thrombocytosis include 
acute or chronic infection or inflammation, iron deficiency, hemolytic anemia, asplenia, and cancer [8].

A multitude of evidence links platelets with cancer pathogenesis $[5,7]$. For example, platelets, reacting to the modified tumor vasculature, can release growth factors or enzymes that stimulate cancer cell proliferation or angiogenesis or degrade extracellular matrix [7]. Additionally, platelets and their released mediators such as cytokines can contribute to the regulation of tumor associated inflammatory reactions [7]. Thrombocytosis is common in CRC, and three recent metaanalyses, based on 30 studies [9], 16 studies [10], and 9 studies [11] indicate that elevated preoperative platelet count is associated with shorter overall and diseasefree survival in CRC. However, comparative analyses including additional prognostic parameters such as lymphatic invasion would be required to establish elevated platelet count as a relevant prognostic indicator in CRC.

Aspirin is one of the most commonly used drugs, with role as analgesic, antipyretic, and agent for cardiovascular prophylaxis [12]. The cardioprotective effects of aspirin are thought to be primarily based on inhibition of platelet production of TXA2 (thromboxane A2) [13], resulting in reduced platelet activation and aggregation. Substantial evidence from observational studies and randomized controlled trials support the efficacy of aspirin in the prevention of cancer, especially CRC [12, 14, 15]. Moreover, aspirin use has been associated with improved outcome in CRC $[12,16]$. Earlier studies have indicated that the benefit of aspirin may be related to the tumor characteristics, such as PTGS2 expression [16] or PIK3CA mutation [17], but also platelet-related mechanisms of action have been suggested [12]. Thus, we hypothesized that the beneficial survival effect of aspirin in CRC could be stronger for patients with high platelet counts.

Colorectal cancer can elicit an anti-tumor immune response that restricts tumor growth and is associated with improved survival [18-20]. However, a systemic inflammatory response to cancer is associated with adverse outcome and may facilitate cancer progression by recruiting or mobilizing tumor-promoting inflammatory cells, attenuating the anti-tumor immune response, enhancing tumor cell migration and circulating tumor cell survival, and modifying the parenchyma of the premetastatic sites [21]. We have previously shown that CRC patients have increased serum levels of IL-6, IL-7, IL-8, and platelet-derived growth factor BB (PDGF-BB), and the patients with distant metastasis have higher serum levels of IL-1ra, IL-4, IL-6, IL-7, IL-8, MCP-1, and PDGF$\mathrm{BB}$ [22]. Although platelets are considered important regulators of tumor associated inflammatory reactions $[7,21]$, the relationships between platelet count, serum cytokine milieu, and tumor infiltrating immune cells in CRC are currently poorly understood.

In this study, we analyzed blood platelet counts in a prospectively recruited cohort of 356 CRC patients and studied its relationships with patient characteristics including aspirin use; markers of systemic inflammation (modified Glasgow Prognostic Score, mGPS; serum levels of CRP, albumin, and 13 cytokines) and blood hemoglobin levels; tumor characteristics including five types of tumor infiltrating immune cells (CD3, CD8, FoxP3, Neutrophil elastase, mast cell tryptase); and survival.

\section{Methods \\ Patients}

This study is based on an earlier described prospectively recruited cohort of surgically treated stage I-IV CRC patients, operated in Oulu University Hospital in 2006-2014 ( $\mathrm{n}=356$; Additional file 1: Table S1) [22-24]. All the patients were required to sign a written informed consent to participate. The study was approved by the Ethics Committee of Oulu University Hospital (58/2005, 184/2009) and was performed in accordance with the Declaration of Helsinki. The clinical details, including the list of patients' medication, were collected from the clinical records and follow-up data from the clinical records and from Statistics Finland [25, 26]. Time to recurrence (TTR) was defined as time from the operation to the recurrence of the same cancer, cancer specific survival (CSS) was defined as time from the operation to death from the same cancer, and overall survival (OS) was defined as time from the operation to death, irrespective of cause. Preoperative staging of rectal cancer was conducted with magnetic resonance imaging, and most rectal cancer patients with cT3 or cT4 tumors $(n=69)$ received preoperative radiotherapy or chemoradiotherapy (RT/CRT). Additionally, one colon cancer patient received neoadjuvant treatment. The study was designed in accordance with the REMARK criteria [27].

\section{Blood analyses}

Blood and serum samples were collected preoperatively [22]. Blood platelet count, blood Hb, erythrocyte MCV, serum CRP and serum albumin were measured in the laboratory of Oulu University Hospital [22, 28]. Anemia was defined according to WHO criteria as blood $\mathrm{Hb}$ levels $<120 \mathrm{~g} / \mathrm{L}$ in women and $<130 \mathrm{~g} / \mathrm{L}$ in men [23]. It was classified according to erythrocyte MCV levels as microcytic $(\mathrm{MCV}<80 \mathrm{fL})$, normocytic (MCV 80-100 fL), and macrocytic $(\mathrm{MCV}>100 \mathrm{fL})$ [23]. CRP and albumin were used to calculate modified Glasgow Prognostic Score (mGPS): mGPS0, serum CRP $\leq 10 \mathrm{mg} / \mathrm{L}$ and serum albumin $\geq 35 \mathrm{~g} / \mathrm{L}$ or $<35 \mathrm{~g} / \mathrm{L} ; \mathrm{mGPS} 1$, serum $\mathrm{CRP}>10 \mathrm{mg} / \mathrm{L}$ and serum albumin $\geq 35 \mathrm{~g} / \mathrm{L} ; \mathrm{mGPS}$, 
serum CRP $>10 \mathrm{mg} / \mathrm{L}$ and serum albumin $<35 \mathrm{~g} / \mathrm{L}$ ) [18, 29]. In patients operated between 2006 and January $2010(n=148)$, serum analysis of 27 cytokines was performed with Bio-Plex Pro Human 27-Plex Cytokine Panel (Bio-Rad, Hercules, CA, USA), as described earlier [22]. As described earlier in more detail, 14 cytokines had many values outside the assay detection limits, and 13 cytokines (IL-1ra, IL-4, IL-6, IL-7, IL-8, IL-9, IL-12, IFN$\gamma$, CXL10, CCL2, CCL4, CCL11, and PDGF-BB) with less than four values outside the assay working range were included in this study $[22,30]$.

\section{Histopathological analysis}

Histopathological analysis was conducted using the hematoxylin and eosin stained sections. Tumor stage was reclassified according to TNM8, and the grading was conducted according to the WHO criteria. Lymphatic invasion was defined as tumor cells present in vessels with an endothelial lining but lacking a muscular wall, while blood vessel invasion was defined as tumor cells in vessels with a thick muscular wall or in vessels containing red blood cells [31].

\section{Immunohistochemistry}

Tissue microarrays were constructed for immunohistochemical analysis, consisting of 1-4 cores of $3.0 \mathrm{~mm}$ diameter for each tumor, depending on the size of the tumor, from the invasive margin (IM) and the tumor center (CT) [23, 32]. Immunohistochemistry for mismatch repair (MMR) enzymes MLH1, MSH2, MSH6, and PMS2 was conducted, as described earlier, to evaluate MMR enzyme status [23, 28, 33]. BRAF V600E specific VE1 immunohistochemistry was conducted with Ventana Bench-Mark XT immunostainer (Ventana Medical Systems, Tucson, AZ) [34], to evaluate BRAF mutation status. Our earlier study indicated that the method had a sensitivity of $100 \%$ and a specificity of $99.3 \%$ in detecting BRAF V600E mutation [34]. The densities of five types of tumor infiltrating immune cells at the invasive margin (IM) and center of the tumor (CT) were determined with an ImageJ based computer assisted analysis method [35], as described earlier $[24,36]$. Intraepithelial (IEL) $\mathrm{CD}^{+}$ and $\mathrm{CD}^{+}$lymphocytes were counted manually from the captured images, because the method could not segregate intraepithelial cells from those in tumor stroma [36]. The antibodies and protocols for immunohistochemistry are specified in Additional file 1: Table S2. All the histological and immunohistochemical analyses were performed blinded to other data.

\section{Statistical analyses}

The statistical analyses were conducted using $\mathrm{R}$ version 3.5.1 (R Foundation for Statistical Computing, Vienna,
Austria), using the packages tidyverse (v.1.2.1), survival (v.2.42-6), survminer (v.0.4.3), plotROC (v.2.2.1), car (v.3.0-2), lm.beta (v.1.5-1), and powerSurvEpi (v.0.1.0), or IBM SPSS Statistics for Windows, version 24.0 (IBM Corp., Armonk, NY). The statistical significances of the associations between categorical and continuous variables were analyzed by independent samples t-test or Mann-Whitney test (comparing two classes), or one-way analysis of variances (ANOVA) or Kruskal-Wallis test (comparing three or more classes), as appropriate, while the statistical significances of the associations between two categorical variables were analyzed with $\chi^{2}$ test. Pearson correlation coefficients $(r)$ were used to determine the correlation between two continuous variables. To normalize their distribution, logarithmic transformation was applied to variables with positive skewness. Multiple linear regression models were used in multivariable analysis of the associations between blood platelet count, systemic inflammatory markers/tumor infiltrating immune cells, and selected clinicopathological factors. The normality of the residuals, homoscedasticity, and linearity assumptions were checked by a histogram and a normal probability plot of the residuals and by a scatterplot of residuals vs. predicted values. Cytoscape, an open source software platform for visualizing complex networks, was used in creating a $2 \mathrm{D}$ visualization of the relationships between blood platelet count, blood Hb levels, and serum levels of systemic inflammatory markers with the Prefuse force directed algorithm weighted by the statistical significances of the correlations between individual variables [37]. Receiver operating characteristics (ROC) analysis was used to evaluate the capacity of platelet count to discriminate the survivors from non-survivors. The survival outcomes of the patient subgroups were analyzed with Kaplan-Meier curves, log-rank test, and Cox proportional hazards regression analysis. In multivariable Cox models, the cases with missing data were assigned to the majority category of a given covariate to limit the degrees of freedom of the models. We confirmed that excluding cases with missing data on any of the covariates did not substantially alter the results (data not shown). An interaction between platelet count and aspirin use was assessed by including the product interaction term of these variables in the model. All p values are two-tailed. As our primary hypothesis testing, we evaluated the relationships between blood platelet count and TTR, CSS, and OS. For these primary, confirmatory survival analyses, we used $\alpha$ level of 0.05 , while for the other exploratory analyses, we used $\alpha$ level of 0.005 [38], and regarded the results with $p=0.05-0.005$ as of borderline statistical significance and interpreted them cautiously. Based on the most recent meta-analysis, we expected to observe a HR of 2.11 for the association between high platelet 
count and OS [11]. The statistical power $(1-\beta)$ to detect this HR was estimated to be 0.98 at $\alpha$ level of $0.05,0.92$ at $\alpha$ level of $0.01,0.88$ at $\alpha$ level of 0.005 , and 0.76 at $\alpha$ level of 0.001 (Additional file 1: Figure S1).

\section{Results}

\section{Platelet count and clinicopathological features}

Among the 356 CRC patients, mean platelet count was $294.9 \times 10^{9} / \mathrm{L}\left(\mathrm{SD} 93.4 \times 10^{9} / \mathrm{L}\right)$ (Table 1$)$. High platelet count, studied as a continuous variable, was associated with female gender $(\mathrm{p}=0.002)$, proximal tumor location $(p<0.001)$, high TNM stage $(p<0.001)$, especially high T-class $(p=0.003)$ and M-class $(p=0.001)$, and normocytic and microcytic anemia $(\mathrm{p}<0.001)$. High platelet count also showed tendency towards an association (borderline statistical significance considering multiple hypothesis testing) with young patient age $(\mathrm{p}=0.044)$ and MMR deficiency $(\mathrm{p}=0.006)$. Platelet count had no association with aspirin use $(\mathrm{p}=0.396)$.

\section{Platelet count and systemic inflammation}

We hypothesized that elevated platelet count would be associated with systemic inflammation in CRC. Supporting the hypothesis, increased platelet count, as a continuous variable, was associated with higher mGPS ( $<<0.001$; Table 1) and with higher serum $C$-reactive protein levels (univariable $\mathrm{p}<0.001$; multivariable adjusted $\mathrm{p}=0.003$; Additional file 1: Table S3).

For more detailed overview on the relationships between platelet count and systemic inflammatory mediators, serum analysis of 13 cytokines was conducted in 148 patients. This analysis indicated that platelet count positively correlated with most of the studied cytokines, including IL-1RA, IL-4, IL-6, IL-7, IL-8, IL-12. IFN and PDGF-BB (univariable and multivariable adjusted $\mathrm{p}<0.001$ for all; Fig. 1; Table 2); the strongest correlations were with IL-7 $(r=0.531)$, IL-1RA $(r=0.509)$, and PDGF-BB $(r=0.474)$.

\section{Platelet count and tumor infiltrating immune cells}

The strong associations between platelet count and serum cytokine levels lead us to hypothesize that platelet count would also contribute to tumor immune cell infiltration by the release of inflammatory mediators. Thus, correlations between platelet count and five types of tumor infiltrating immune cells $\left(\mathrm{CD}^{+} \mathrm{T}\right.$ cells, $\mathrm{CD} 8^{+}$ cytotoxic $\mathrm{T}$ cells, FoxP3 ${ }^{+}$regulatory $\mathrm{T}$ cells, Tryptase ${ }^{+}$ mast cells, Elastase ${ }^{+}$neutrophils) in different tumor locations were analyzed (Fig. 2; Table 3). However, no statistically significant multivariable adjusted associations were detected between platelet count and the analyzed cell types.
Table 1 Relationships between blood platelet count and clinicopathological characteristics

\begin{tabular}{|c|c|c|}
\hline Variable & $\begin{array}{l}\text { Blood platelet } \\
\text { count }\left(10^{9} / L\right) \text {, mean } \\
\text { (sd) }\end{array}$ & $\mathrm{p}$ value \\
\hline All patients, $n=356$ & $294.9(93.4)$ & \\
\hline \multicolumn{3}{|l|}{ Age } \\
\hline$<65, n=130$ & $308.0(94.0)$ & \multirow[t]{2}{*}{0.044} \\
\hline$=65, n=226$ & $287.4(92.4)$ & \\
\hline \multicolumn{3}{|l|}{ Gender } \\
\hline Male, $n=190$ & $280.9(97.3)$ & \multirow[t]{2}{*}{0.002} \\
\hline Female, $n=166$ & $310.9(86.3)$ & \\
\hline \multicolumn{3}{|l|}{ Body mass index } \\
\hline$<25, n=123$ & $298.7(102.2)$ & \multirow[t]{3}{*}{0.688} \\
\hline $25-30, n=146$ & $288.8(81.5)$ & \\
\hline$>30, n=78$ & $293.5(100.5)$ & \\
\hline \multicolumn{3}{|l|}{ Tumor location } \\
\hline Proximal colon, $\mathrm{n}=123$ & $319.4(90.6)$ & \multirow[t]{3}{*}{$<0.001$} \\
\hline Distal colon, $n=73$ & $301.7(102.2)$ & \\
\hline Rectum, $n=160$ & $273.0(86.4)$ & \\
\hline \multicolumn{3}{|c|}{ Neoadjuvant therapy in rectal cancer patients } \\
\hline No, $n=91$ & $279.0(95.7)$ & \multirow[t]{2}{*}{0.295} \\
\hline Yes, $n=69$ & $265.0(72.4)$ & \\
\hline \multicolumn{3}{|l|}{ WHO grade } \\
\hline Low-grade $(1-2), n=308$ & $293.1(93.2)$ & \multirow[t]{2}{*}{0.406} \\
\hline High-grade (3), $n=46$ & $305.4(96.3)$ & \\
\hline \multicolumn{3}{|l|}{ Stage } \\
\hline Stage $\mathrm{l}, \mathrm{n}=81$ & $275.0(69.6)$ & \multirow[t]{4}{*}{$<0.001$} \\
\hline Stage $\|, n=113$ & $296.8(91.8)$ & \\
\hline Stage $I I I, n=116$ & $283.5(87.6)$ & \\
\hline Stage $I V, n=45$ & $351.2(122.0)$ & \\
\hline \multicolumn{3}{|l|}{ T class } \\
\hline $\mathrm{T} 1, \mathrm{n}=15$ & $271.4(81.7)$ & \multirow[t]{4}{*}{0.003} \\
\hline $\mathrm{T} 2, \mathrm{n}=89$ & $270.6(64.5)$ & \\
\hline$T 3, n=225$ & $299.1(95.0)$ & \\
\hline $\mathrm{T} 4, \mathrm{n}=26$ & $355.8(134.4)$ & \\
\hline \multicolumn{3}{|l|}{$N$ class } \\
\hline No, $n=201$ & $289.2(84.8)$ & \multirow[t]{3}{*}{0.124} \\
\hline$N 1, n=96$ & $291.5(98.0)$ & \\
\hline$N 2, n=57$ & $317.4(109.5)$ & \\
\hline \multicolumn{3}{|l|}{ M class } \\
\hline $\mathrm{M0}, \mathrm{n}=311$ & $286.8(85.7)$ & \multirow[t]{2}{*}{0.001} \\
\hline$M 1, n=45$ & $351.2(122.0)$ & \\
\hline \multicolumn{3}{|l|}{ Lymphatic invasion } \\
\hline No, $n=192$ & $287.9(85.7)$ & \multirow[t]{2}{*}{0.162} \\
\hline Yes, $n=160$ & $302.1(101.4)$ & \\
\hline \multicolumn{3}{|l|}{ Blood vessel invasion } \\
\hline No, $n=293$ & $291.4(89.3)$ & \multirow[t]{2}{*}{0.263} \\
\hline Yes, $n=59$ & $308.7(110.8)$ & \\
\hline Mismatch repair enzyme sta & & \\
\hline Proficient, $n=315$ & $290.1(92.4)$ & 0.006 \\
\hline Deficient, $n=40$ & $332.9(94.9)$ & \\
\hline
\end{tabular}


Table 1 (continued)

\begin{tabular}{lll}
\hline Variable & $\begin{array}{l}\text { Blood platelet } \\
\text { count (109/L), mean } \\
\text { (sd) }\end{array}$ & p value \\
\hline BRAFVE1 immunohistochemistry & & \\
$\quad$ Negative, $n=322$ & $292.7(93.8)$ & 0.126 \\
Positive, $n=33$ & $318.8(87.3)$ & \\
Modified Glasgow Prognostic Score & & \\
mGPSO, $n=269$ & $283.1(86.3)$ & $<0.001$ \\
mGPS1, $n=63$ & $335.7(101.5)$ & \\
mGPS2, $n=8$ & $413.2(148.2)$ & \\
Anemia & & \\
No, $n=202$ & $264.5(73.7)$ & \\
Yes, $n=154$ & $334.8(101.4)$ & \\
Anemia category & & \\
No anemia, $n=202$ & $264.5(73.7)$ & \\
Microcytic anemia, $n=43$ & $372.8(113.4)$ & \\
Normocytic anemia, $n=109$ & $322.6(91.5)$ & \\
Macrocytic anemia, $n=2$ & $181.0(58.0)$ & \\
Aspirin use & & \\
No, $n=271$ & $297.3(90.5)$ & \\
Yes, $n=85$ & &
\end{tabular}

\section{Aspirin use and colorectal cancer characteristics}

We hypothesized that the effects of platelets in CRC could depend on whether patients receive aspirin medication inhibiting platelet activation. Therefore, we analyzed the relationships between aspirin use, clinicopathological characteristics (Additional file 1: Table S4), systemic inflammatory markers (Additional file 1: Table S5), and tumor infiltrating immune cells (Additional file 1: Table S5). There were 85 (23.9\%) patients, who had aspirin in their list of daily medications in the clinical records at the time of surgery, with dosage of $100 \mathrm{mg} /$ day $(\mathrm{n}=75,88.2 \%)$ or $50 \mathrm{mg} /$ day $(\mathrm{n}=10,11.8 \%)$. Aspirin use was more prevalent in the older age group $(\mathrm{p}<0.001)$, among the patients who did not receive preoperative RT/CRT ( $\mathrm{p}=0.010$; borderline statistical significance considering multiple hypothesis testing), in lower $\mathrm{N}$-class $(\mathrm{p}=0.026$; borderline statistical significance considering multiple hypothesis testing), and in patients with anemia ( $p=0.039$; borderline statistical significance considering multiple hypothesis testing). Aspirin use did not significantly associate with systemic inflammatory markers (Additional file 1: Table S6) but associated with a tendency towards higher numbers of FoxP3 ${ }^{+} \mathrm{T}$ cells at the IM $(\mathrm{p}=0.005)$ and higher numbers of neutrophils in the CT $(p=0.031$; borderline statistical significance considering multiple hypothesis testing).

\section{Survival analyses}

Finally, survival analyses were conducted. ROC analysis indicated that blood platelet count did not show good ability to discriminate survivors from non-survivors (Fig. $3 \mathrm{a}-\mathrm{c}$ ). In the previous studies, $400 \times 10^{9} / \mathrm{L}$ has been the most commonly used cut-off for high platelet count [11], and we decided to apply that to our primary analyses. However, subsequent Kaplan-Meier curves (Fig. 3df), and univariable Cox regression models (Additional file 1: Table S6) did not indicate statistically significant association between platelet count and survival. To evaluate the consistency of our data with different cutoff values, we also tested $300 \times 10^{9} / \mathrm{L}$ and $200 \times 10^{9} / \mathrm{L}$ as the cut-off points, and the results were parallel to those with $400 \times 10^{9} /$ L (Additional file 1: Figs. S2, S3, Table S6). We hypothesized that aspirin use could influence the prognostic effect of platelet count. However, combined evaluation of platelet count and aspirin did not show statistically significant associations with survival (Fig. 3g-i, Additional file 1: Figs. S2, S3, Table S6). Blood platelet count and aspirin use were not statistically significant in multivariable Cox models (Table 4). There was no statistically significant interaction between platelet count and aspirin use (TTR: $p$ for interaction $=0.933$, CSS: $\mathrm{p}$ for interaction $=0.536$, OS: $\mathrm{p}$ for interaction $=0.500$ ).

\section{Discussion}

Platelets have been implicated with an important role in cancer pathogenesis, especially in regulating inflammatory reactions. The main findings of this study indicate that high platelet count is associated with elevated systemic inflammatory markers in CRC patients. Platelet count, however, was not statistically significantly associated with the density of tumor-infiltrating immune cells or CRC patient survival.

Systemic inflammation can facilitate tumor progression and metastasis [21]. One of the best validated systemic inflammatory prognostic markers, mGPS is comprised of serum CRP and serum albumin [29,39]. The results of this study indicate that high platelet count is associated with elevated mGPS and serum CRP levels, supporting the role of thrombocytosis in cancer associated systemic inflammation.

For a more detailed analysis of the relationships between platelet count and systemic inflammatory regulators, we measured serum levels of 13 cytokines. We utilized Cytoscape, an open source software platform for visualizing complex networks [37], in creating a $2 \mathrm{D}$ visualization of the relationships between blood platelet count and serum cytokine levels, since visual information can facilitate the comprehension of large quantities of correlation data [40]. The analysis showed 


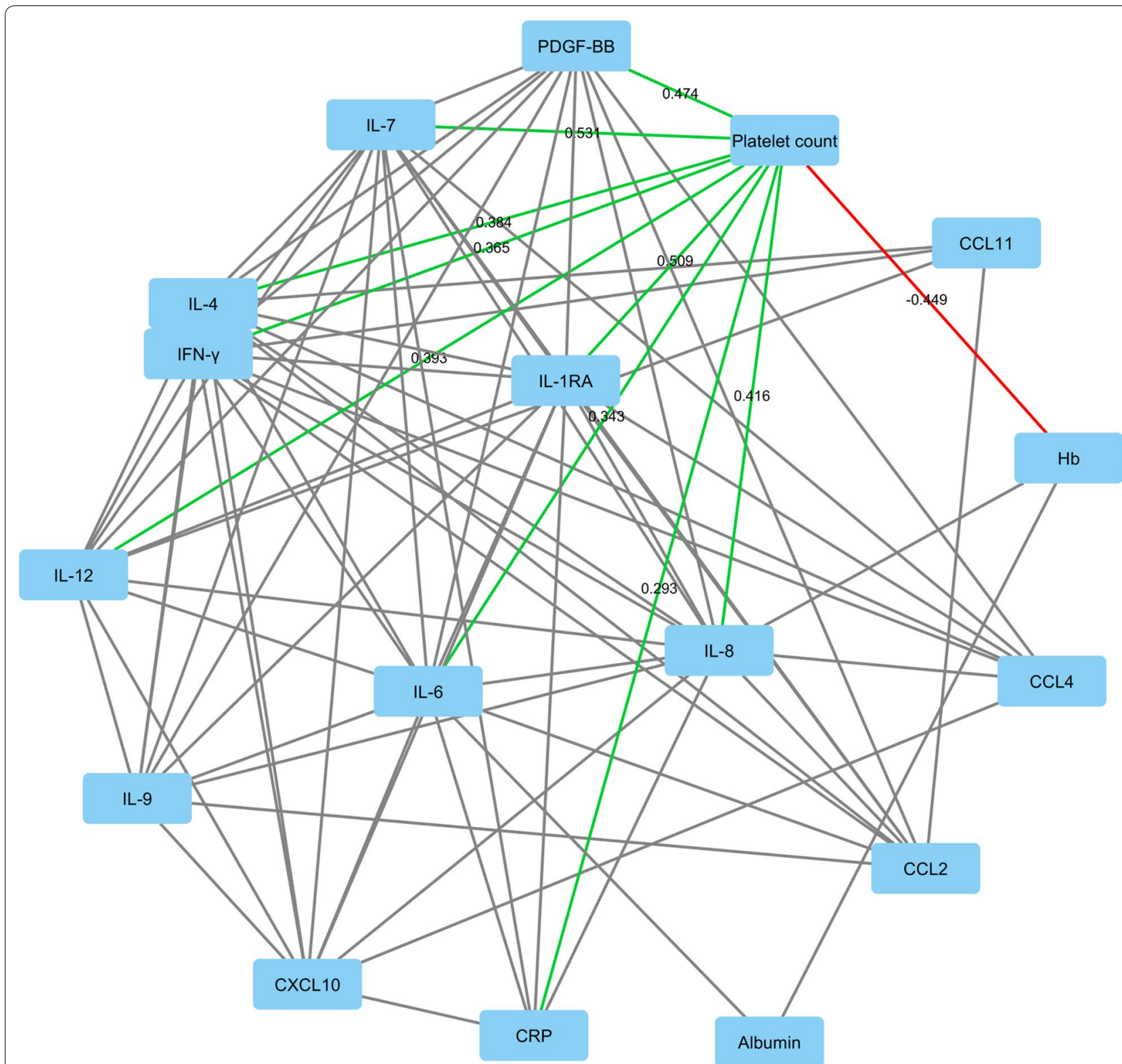

Fig. 1 2D visualization of the relationships between blood platelet count, blood hemoglobin levels, serum C-reactive protein levels, serum albumin levels, and serum cytokine levels in a subset of 148 patients. The edges (connecting lines) depict the associations between the variables (only those with $p<0.001$ shown). The edge length illustrates the significance of the association. The correlations between platelet count and other variables are represented by green (positive correlation) and red (negative correlation) edges, with the label indicating corresponding Pearson $r$ for the correlation. The other associations are indicated by the grey edges. The 2D visualization was created with Cytoscape software platform [37], utilizing the Prefuse force directed algorithm weighted by the statistical significances of the correlations between individual variables. CCL: $C$ hemokine $(C-C$ motif) ligand; CRP: C-reactive protein; CXCL: chemokine (C-X-C motif) ligand; Hb: hemoglobin; IFN: interferon; IL: interleukin; PDGF: platelet-derived growth factor

that there were strong positive correlations between platelet count and multiple serum cytokine levels, most notably with IL-7, IL-1RA, and PDGF-BB. The mechanisms underlying the observed correlations between platelet count and serum cytokines were not addressed in our study. Thus, these correlations may indicate that (a) platelets contribute to the production of these cytokines or store and release these cytokines, (b) these cytokines contribute to the increased platelet production, or (c) some other unrecognized factors such as shared background factors are involved. 
Table 2 Correlation between blood platelet count and serum cytokine levels

\begin{tabular}{|c|c|c|c|c|c|}
\hline \multirow[t]{2}{*}{ Variable } & \multirow{2}{*}{$\begin{array}{l}\mathrm{N} \\
\text { (unadjusted, } \\
\text { adjusted) }\end{array}$} & \multicolumn{2}{|c|}{ Unadjusted } & \multicolumn{2}{|c|}{ Adjusted } \\
\hline & & Pearson $r$ & $p$ value & Beta & $\mathrm{p}$ value \\
\hline IL-1RA & 148,146 & 0.509 & $<0.001$ & 0.428 & $<0.001$ \\
\hline IL-4 & 148,146 & 0.384 & $<0.001$ & 0.354 & $<0.001$ \\
\hline IL-6 & 147,145 & 0.343 & $<0.001$ & 0.265 & $<0.001$ \\
\hline IL-7 & 148,146 & 0.531 & $<0.001$ & 0.521 & $<0.001$ \\
\hline IL-8 & 148,146 & 0.416 & $<0.001$ & 0.286 & $<0.001$ \\
\hline IL-9 & 147,145 & 0.243 & 0.003 & 0.237 & 0.002 \\
\hline IL-12 & 148,146 & 0.393 & $<0.001$ & 0.363 & $<0.001$ \\
\hline IFNY & 148,146 & 0.365 & $<0.001$ & 0.317 & $<0.001$ \\
\hline CXCL10 & 148,146 & 0.156 & 0.058 & 0.107 & 0.158 \\
\hline CCL2 & 147,145 & 0.146 & 0.078 & 0.150 & 0.052 \\
\hline CCL4 & 148,146 & 0.159 & 0.054 & 0.094 & 0.213 \\
\hline CCL11 & 148,146 & 0.004 & 0.959 & 0.094 & 0.212 \\
\hline PDGF & 148,146 & 0.474 & $<0.001$ & 0.464 & $<0.001$ \\
\hline
\end{tabular}

Blood platelet count and serum cytokines were logarithmically transformed because of positive skewness. The adjusted correlations were calculated with multiple linear regression. The correlations were adjusted for tumor location (colon vs. rectum), preoperative radiotherapy or chemoradiotherapy, tumor stage variables (T1-2 vs. T3-4; N0 vs. N1-2; M0 vs. M1), patient age, patient gender, and blood hemoglobin levels

CCL: chemokine ( $\mathrm{C}-\mathrm{C}$ motif) ligand; $\mathrm{CXCL}$ : chemokine (C-X-C motif) ligand; IFN: interferon; IL: interleukin; PDGF: platelet-derived growth factor

Platelet granules are packed with enzymes, growth factors and cytokines, which are released on platelet activation [41]. Accordingly, the concentrations of many cytokines in plasma and serum samples are not equal. For example, among the cytokines showing high correlation with platelet count in this study, IL-7 and PDGF-BB are found in platelet granules $[41,42]$. A recent study utilized the same Bio-Rad cytokine panel that was used in this study to compare the cytokine levels in serum and plasma of healthy subjects [43]. The results indicated that the levels of PDGF-BB, IL-4, IL-7, and CXCL10 were significantly higher in serum samples compared to heparin plasma, suggesting that these cytokines are released from platelets during clotting. However, the same study did not show statistically significant difference in serum levels of IL-1RA, IL-6, IL-8, IL-9, IL-12, IFN- $\gamma$, CCL2, CCL4, and CCL11, relative to heparin plasma levels. Of the cytokines potentially released from platelet granules, IL-7 is a major regulator of T cell homeostasis, whereas its role in malignancy is controversial [44]. PDGF-BB exerts growth factor functions during embryonal development and adult tissue homeostasis and repair such as wound healing, whereas in cancer, this molecule can directly stimulate the growth and survival of tumor cells and tumor stromal cells [45]. IL-4 is an anti-inflammatory cytokine, which can also contribute to the survival of CRC stem cells [46]. Thus, the particles released by platelets can contribute to the regulation of tumor growth and tumor associated inflammatory reactions.

Thrombopoietin is regarded as a key hormone in megakaryocyte differentiation and proliferation, resulting in platelet production [8]. The process is closely regulated, since thrombopoietin in plasma binds to the circulating platelet surface receptors and only the remaining free thrombopoietin is available to promote megakaryocyte proliferation [8]. Hepatocytes are a major source of thrombopoietin, and inflammation, particularly IL-6, increases thrombopoietin production [8]. Accordingly, we observed positive correlation between platelet count and serum IL-6 levels, potentially reflecting the up-regulation of thrombopoietin production caused by increased availability of IL-6 in CRC. Notably, our earlier study has indicated that IL-6 is among the cytokines showing the highest increase in CRC, as well as the strongest association with high tumor stage [22]. Thus, thrombocytosis is one of the potential systemic consequences of cancerinduced liver-reprogramming. The potential effects of cytokines on platelets are not limited to platelet production, since a recent study indicated that the presence of cytokines such as IL-6 and IL-8, both of which show positive correlation with platelet count in our data, can also alter platelet structure causing platelet hyperactivation [47].

An anti-tumor immune response can control cancer growth [48], and increased density of tumor infiltrating lymphocytes has been associated with improved survival in CRC $[18,19]$. We hypothesized that, by the release of soluble inflammatory mediators, platelets could contribute to the regulation of tumor immune cell infiltration. However, no statistically significant multivariable adjusted correlations were observed between platelet count and the densities of tumor infiltrating $\mathrm{CD}^{+}$, $\mathrm{CD}^{+}$, and FoxP3 ${ }^{+} \mathrm{T}$ cells, neutrophils, or mast cells. This suggests that platelet count only account for a minority of the changes in the densities of tumor infiltrating immune cells, while other factors such as microsatellite instability associated with increased tumor immunogenicity are more important $[49,50]$.

Improved prognostic parameters are needed to classify CRC into more homogenous and therapeutically relevant groups. Three recent meta-analyses [9-11] suggest that elevated preoperative platelet count is associated with decreased survival in CRC. In our present study, higher platelet counts were observed in higher tumor stage, but platelet count was not significantly associated with survival. Thus, the results do not support the prognostic value of platelet count in unselected stage I-IV CRC patients. Our sample size did not enable comprehensible evaluation of the effect of platelet count in more specific patient subgroups, such as stage II patients. Relative to 


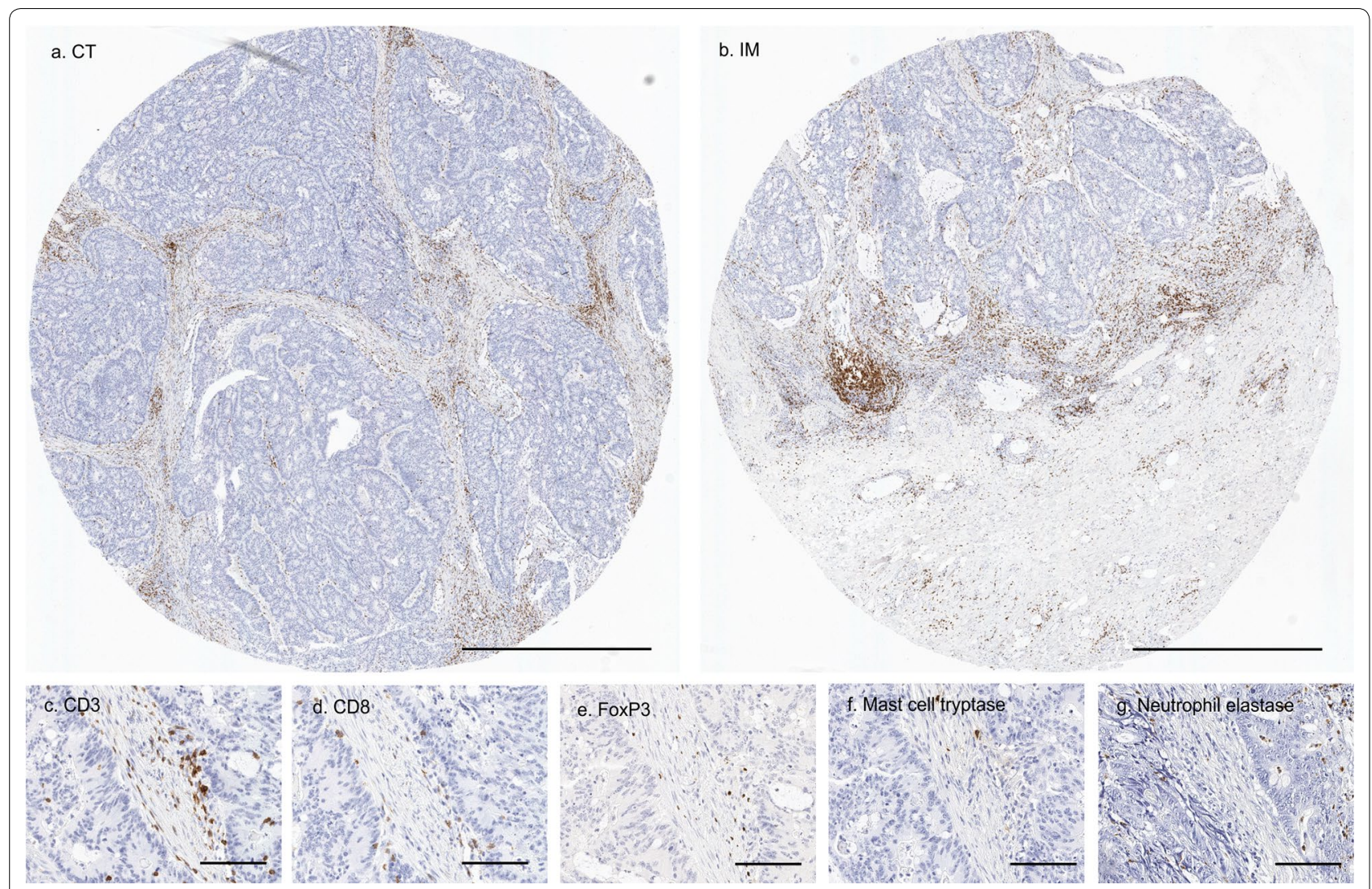

Fig. 2 Detection of five types of immune cells in colorectal cancer tissue microarrays with immunohistochemistry. $\mathbf{a}, \mathbf{b}$ Examples of tissue microarray cores from the center of the tumor (CT) and the invasive margin (IM) with CD3 immunohistochemistry. Scale bar is $1 \mathrm{~mm}$. c-g Close-up views portraying $\mathrm{CD}^{+} \mathrm{T}$ cells, $\mathrm{CD} 8^{+}$cytotoxic T cells, FoxP3 ${ }^{+}$regulatory T cells, Tryptase ${ }^{+}$mast cells, and Elastase ${ }^{+}$neutrophils. Scale bar is $100 \mu \mathrm{m}$

Table 3 Correlations between blood platelet count and the densities of tumor infiltrating immune cells

\begin{tabular}{|c|c|c|c|c|c|}
\hline \multirow[t]{2}{*}{ Variable } & \multirow[t]{2}{*}{$\mathrm{N}$ (unadjusted, adjusted) } & \multicolumn{2}{|c|}{ Unadjusted } & \multicolumn{2}{|c|}{ Adjusted } \\
\hline & & Pearson $r$ & $p$ value & Beta & $p$ value \\
\hline CD3 IM & 354,352 & 0.035 & 0.511 & 0.033 & 0.503 \\
\hline $\mathrm{CD} 3 \mathrm{CT}$ & 355,353 & -0.021 & 0.694 & 0.015 & 0.761 \\
\hline CD3 IEL & 352,350 & 0.058 & 0.280 & 0.029 & 0.565 \\
\hline CD8 IM & 355,353 & 0.097 & 0.068 & 0.057 & 0.272 \\
\hline $\mathrm{CD} 8 \mathrm{CT}$ & 355,353 & -0.017 & 0.752 & -0.032 & 0.513 \\
\hline CD8 IEL & 345,344 & 0.062 & 0.247 & 0.033 & 0.519 \\
\hline FoxP3 IM & 354,352 & -0.055 & 0.301 & -0.007 & 0.899 \\
\hline FoxP3 CT & 354,352 & -0.108 & 0.043 & -0.054 & 0.281 \\
\hline Mast cell tryptase IM & 354,352 & -0.119 & 0.025 & -0.054 & 0.255 \\
\hline Mast cell tryptase CT & 354,352 & -0.078 & 0.143 & 0.008 & 0.867 \\
\hline Neutrophil elastase IM & 347,345 & 0.015 & 0.776 & 0.035 & 0.470 \\
\hline Neutrophil elastase CT & 347,345 & 0.042 & 0.435 & 0.000 & 1.000 \\
\hline
\end{tabular}

Blood platelet count and immune cell densities were logarithmically transformed because of positive skewness. The correlations were adjusted for tumor location (colon vs. rectum), preoperative radiotherapy or chemoradiotherapy, tumor stage variables (T1-2 vs. T3-4; N0 vs. N1-2; M0 vs. M1), patient age, patient gender, and blood hemoglobin levels with multiple linear regression

IM: invasive margin; CT: center of tumor; IEL: intraepithelial 

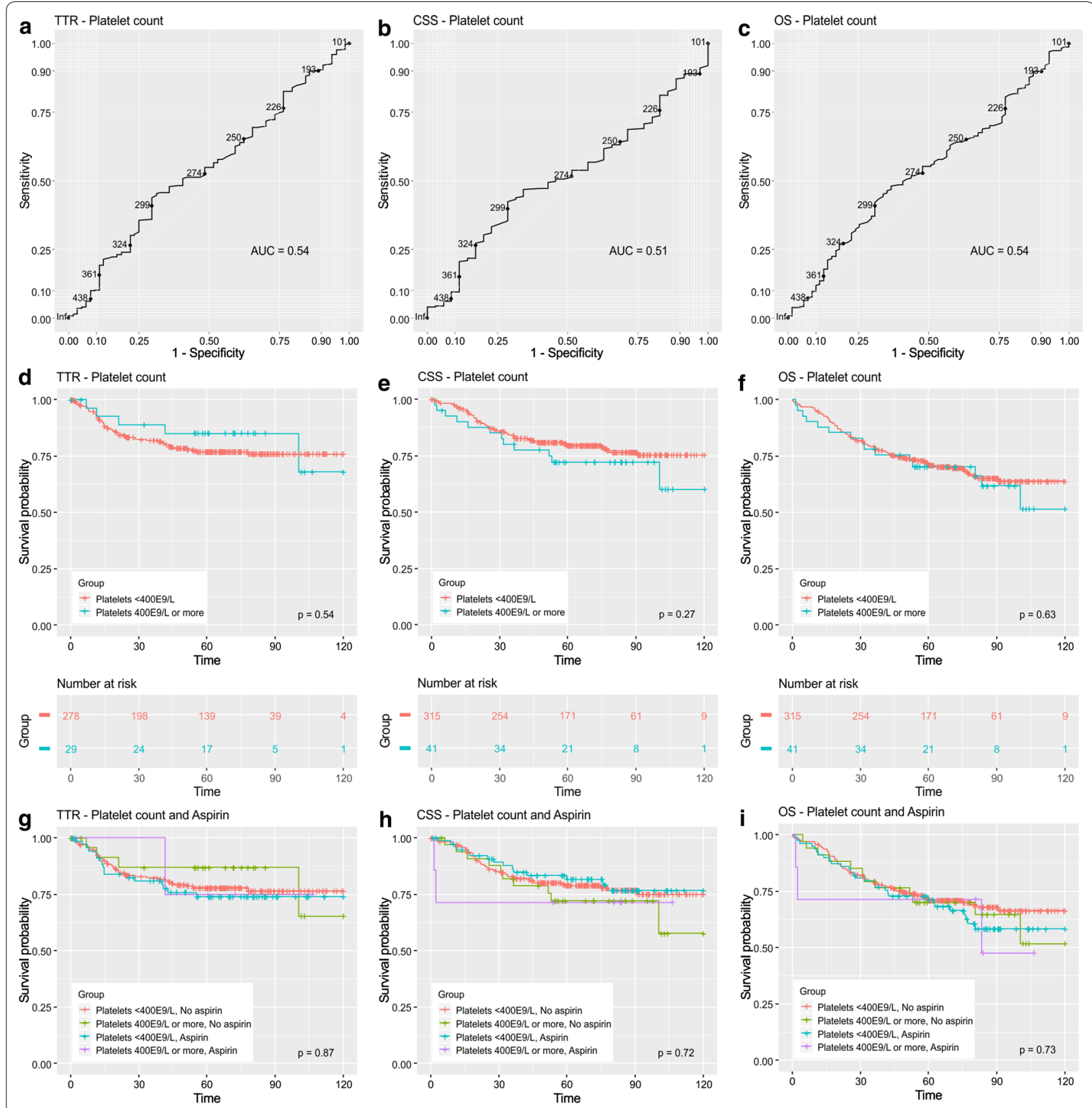

h CSS - Platelet count and Aspirin
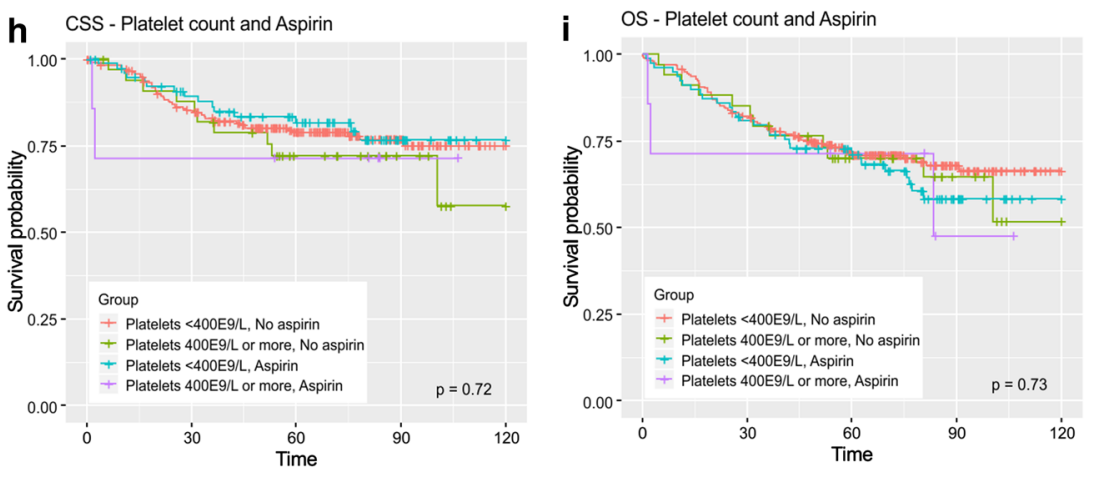

Number at risk

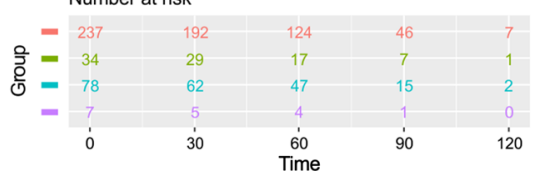

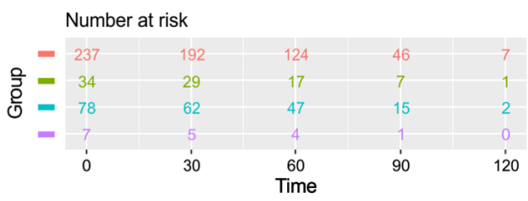

Fig. 3 Platelet count, aspirin use, and colorectal cancer survival. a-c Receiver-operating characteristics (ROC) curves displaying the ability of blood platelet count to distinguish time to recurrence (TTR), cancer specific survival (CSS), and overall survival (OS). d-f Kaplan-Meier curves showing the relationships between blood platelet count and TTR, CSS, and OS. g-i Kaplan-Meier curves showing the relationships between combined classification of blood platelet count and aspirin use and TTR, CSS, and OS 
Table 4 Cox proportional hazard regression models for time to recurrence (TTR), cancer-specific survival (CSS), and overall survival (OS) according to blood platelet count and clinicopathological characteristics

\begin{tabular}{|c|c|c|c|c|c|c|c|c|c|}
\hline & \multicolumn{3}{|l|}{$\mathrm{TTR}^{\mathrm{a}}$} & \multicolumn{3}{|l|}{$\mathrm{CSS}^{\mathbf{b}}$} & \multicolumn{3}{|l|}{ OS $^{c}$} \\
\hline & HR & $95 \% \mathrm{Cl}$ & p value & HR & $95 \% \mathrm{Cl}$ & p value & HR & $95 \% \mathrm{Cl}$ & p value \\
\hline Patient age (<65 vs. $\geq 65)$ & 1.25 & $0.72-2.17$ & 0.428 & 1.90 & $1.12-3.24$ & 0.018 & 1.93 & $1.24-3.00$ & 0.003 \\
\hline Patient gender (male vs. female) & 0.93 & $0.56-1.55$ & 0.794 & 1.45 & $0.90-2.32$ & 0.124 & 1.10 & $0.76-1.61$ & 0.605 \\
\hline Tumor invasion (T1-T2 vs. T3-T4) & 1.86 & $0.91-3.80$ & 0.091 & 1.25 & $0.65-2.39$ & 0.504 & 1.20 & $0.74-1.96$ & 0.456 \\
\hline Nodal metastases (N0 vs. N1-N2) & 4.89 & $2.50-9.57$ & $<0.001$ & 2.66 & $1.40-5.05$ & 0.003 & 2.10 & $1.29-3.39$ & 0.003 \\
\hline Distant metastases (M0 vs. M1) & - & - & - & 6.43 & $3.63-11.4$ & $<0.001$ & 4.61 & $2.80-7.62$ & $<0.001$ \\
\hline Tumor location (colon vs. rectum) & 1.13 & $0.61-2.09$ & 0.697 & 0.86 & $0.51-1.46$ & 0.577 & 0.83 & $0.53-1.31$ & 0.430 \\
\hline $\begin{array}{l}\text { Preoperative radiotherapy or chemo- } \\
\text { radiotherapy (no vs. yes) }\end{array}$ & 1.25 & $0.61-2.53$ & 0.541 & 1.43 & $0.69-2.94$ & 0.334 & 1.36 & $0.75-2.46$ & 0.319 \\
\hline Lymphatic invasion (no vs. yes) & 1.20 & $0.64-2.24$ & 0.572 & 1.89 & $1.01-3.54$ & 0.046 & 1.33 & $0.83-2.14$ & 0.236 \\
\hline mGPS (0 vs. 1-2) & 0.69 & $0.29-1.65$ & 0.402 & 1.72 & $1.00-2.96$ & 0.049 & 1.67 & $1.07-2.61$ & 0.023 \\
\hline Normocytic anemia (no vs. yes) & 0.89 & $0.47-1.67$ & 0.709 & 1.34 & $0.82-2.20$ & 0.246 & 1.49 & $1.00-2.22$ & 0.052 \\
\hline $\begin{array}{l}\text { Blood platelet count }\left(<400 \times 10^{9} / \mathrm{L}\right. \\
\left.\text { vs. } \geq 400 \times 10^{9} / \mathrm{L}\right)\end{array}$ & 0.77 & $0.30-1.99$ & 0.594 & 0.76 & $0.39-1.48$ & 0.418 & 0.68 & $0.38-1.22$ & 0.197 \\
\hline Aspirin (no vs. yes) & 1.22 & $0.68-2.19$ & 0.499 & 0.94 & $0.52-1.68$ & 0.833 & 1.13 & $0.73-1.75$ & 0.590 \\
\hline
\end{tabular}

The models aimed to enlighten the prognostic value of blood platelet count in CRC, relative to patient age and gender, TNM variables, lymphatic invasion, systemic inflammation (mGPS), normocytic anemia, aspirin use

$\mathrm{Cl}$ : confidence interval; CSS: cancer specific survival; HR: hazard ratio; mGPS: modified Glasgow Prognostic Score; OS: overall survival; TTR: time to recurrence

${ }^{a} \mathrm{n}=306$; median follow-up time 60.4 months (IQR 25.0-79.8); 64 (20.9\%) events; 50 (14.0\%) cases excluded from the analysis because the operation was not radical or no follow-up data available

b $\mathrm{n}=356$; median follow-up time 64.5 months (IQR 37.3-85.6); 77 (21.6\%) events

c $\mathrm{n}=356$; median follow-up time 64.5 months (IQR 37.3-85.6); 114 (32.0\%) events

earlier studies, the major advantage of this study was the inclusion of vast number of additional prognostic parameters such as lymphatic and blood vessel invasion, anemia, and mGPS, for comparison. In addition to elevated systemic inflammatory markers, low blood hemoglobin level was one of the best determinants of high platelet count in this study. The association between reactive thrombocytosis and anemia is also well-known in general population but its pathophysiology remains incompletely understood [51]. We observed high platelet count in patients with either normocytic or microcytic anemia. Microcytic anemia is most commonly due to iron deficiency, while chronic inflammatory conditions are among the most common causes of normocytic anemia in general population [52]. In CRC patients, not only normocytic but also microcytic anemia, is associated with systemic inflammation [23, 53]. Both systemic inflammation and anemia have been associated with adverse clinical outcome in CRC $[29,53-55]$, and this study highlights the importance to include them as comparison for platelet count also in the subsequent studies evaluating the prognostic significance of platelet count.

Inhibiting cyclooxygenase enzymes and thus platelet activation, aspirin is frequently used in cardiovascular event prevention [56]. In the setting of cancer, aspirin use has been associated with decreased CRC incidence and improved CRC outcome [12]. We hypothesized that the beneficial effect of aspirin in CRC could be limited to patients with high platelet counts. However, we did not observe significantly improved survival in the patients who received aspirin regardless of the platelet count. Small patient numbers in some subgroups, in particular, in the aspirin treated group with high platelet count, might have contributed to this negative result, increasing the risk of type 2 error. Moreover, our sample size did not enable us to analyze, whether different aspirin dosages $(100 \mathrm{mg} /$ day or $50 \mathrm{mg} /$ day) were differentially associated with tumor or patient characteristics or survival. Thus, additional studies with larger cohorts are warranted. In addition, the aspirin use data was based on medical records, and potential misclassification due to missing data on over the counter aspirin use has to be taken into account.

Also, some other limitations need to be considered in the interpretation of our results. First, tissue microarrays were used in the immunohistochemical analyses. As only a minor fraction of tumor tissue is analyzed, this may not be representative of the whole tissue section. However, numerous studies have generated reproducible results using tissue microarrays [57]. Moreover, our tissue microarrays were optimized for immune cell counting, including large (diameter $3.0 \mathrm{~mm}$ ) cores from different 
parts of the tumor (Fig. 2). An accurate, earlier validated computer assisted immune cell counting method was used [35]. Second, multiple hypotheses were tested in this observational study. However, we adjusted the level of statistical significance of our exploratory analyses to $\mathrm{p}=0.005$ [38] and interpreted the results with $\mathrm{p}=0.05$ 0.005 (considered borderline statistical significance) cautiously. This approach results in some increase in type 2 statistical error but reduce the risk of type 1 error. The advantages were a prospectively recruited study population, with consistent and extensive histological analysis, including additional prognostic parameters such as lymphatic invasion, and systemic inflammatory markers. An assemblage of tumor infiltrating immune cell types and serum cytokines were analyzed, creating a detailed view on the relationships between platelet count and inflammatory markers.

\section{Conclusions}

In conclusion, blood platelet count is associated with systemic inflammation in CRC but not significantly with the densities of tumor infiltrating $\mathrm{T}$ cells. Especially, platelet count and serum cytokine levels are closely related. Our results do not support the prognostic value of platelet count in general stage I-IV material, but further comparative analyses including blood platelet count and $\mathrm{Hb}$ levels, as well as systemic inflammatory markers in more specific patient subgroups, such as stage II patients, are warranted. This study could not demonstrate associations between aspirin use and platelet count, systemic inflammation, or CRC molecular characteristics.

\section{Additional file}

Additional file 1. Additional Tables S1-S6 and Figures S1-S3.

\begin{abstract}
Abbreviations
CCL: chemokine (C-C motif) ligand; Cl: confidence interval; $C R C$ : colorectal cancer; CRP: C-reactive protein; CSS: cancer specific survival; CT: center of tumor; CXCL: chemokine (C-X-C motif) ligand; Hb: hemoglobin; HR: hazard ratio; IFN: interferon; LL: interleukin; IM: invasive margin; mGPS: modified Glasgow Prognostic Score; MMR: mismatch repair; OS: overall survival; PDGF: platelet-derived growth factor; ROC: receiver operating characteristics; RT/ CRT: radiotherapy or chemoradiotherapy; SD: standard deviation; TNM: tumor, node, metastasis; TTR: time to recurrence; WHO: World Health Organization.
\end{abstract}

\section{Acknowledgements}

The authors are grateful for Ms. Riitta Vuento for her expert assistance.

\section{Authors' contributions}

Study conception and design: JPV, AT, MJM. Data collection: JPV, SAV, PS, IM, KK, TK, JM, KHH, TJK, AT, MJM. Statistical analysis: JPV. Manuscript draft: JPV, SAV. Manuscript review and editing: JPV, SAV, PS, IM, KK, TK, JM, KHH, TJK, AT, MJM. All authors read and approved the final manuscript.

\section{Funding}

This work was supported by grants from Emil Aaltonen Foundation and Orion Research Foundation.

\section{Availability of data and materials}

The patients recruited to this study have given their consent that does not allow us to make the data publicly available according to current data protection legislation.

\section{Ethics approval and consent to participate}

The study was conducted under the approval of Ethics Committee of Oulu University Hospital $(58 / 2005,184 / 2009)$. All the experiments were conducted in accordance with the Declaration of Helsinki. The patients had signed a written informed consent to participate in the study.

\section{Consent for publication}

Not applicable.

\section{Competing interests}

The authors declare that they have no competing interests.

\section{Author details}

${ }_{1}^{1}$ Cancer and Translational Medicine Research Unit, University of Oulu, POB 5000, 90014 Oulu, Finland. ${ }^{2}$ Department of Pathology, Oulu University Hospital and Medical Research Center Oulu, POB 21, 90029 Oulu, Finland. ${ }^{3}$ Department of Oncologic Pathology, Dana-Farber Cancer Institute and Harvard Medical School, 450 Brookline Ave, Boston, MA 02215, USA. ${ }^{4}$ Department of Medical Oncology, Dana-Farber Cancer Institute and Harvard Medical School, 450 Brookline Ave, Boston, MA 02215, USA. ${ }^{5}$ Research Unit of Surgery, Anesthesia and Intensive Care, University of Oulu, POB 5000, 90014 Oulu, Finland. ${ }^{6}$ Department of Surgery, Oulu University Hospital and Medical Research Center Oulu, POB 21, 90029 Oulu, Finland. ${ }^{7}$ Research Unit of Biomedicine and Biocenter of Oulu, University of Oulu, POB 5000, 90014 Oulu, Finland. ${ }^{8}$ Oulu University Hospital and Medical Research Center Oulu, POB 21, 90029 Oulu, Finland. ${ }^{9}$ Department of Gastroenterology and Metabolism, Poznan University of Medical Sciences, ul. Szpitalna 27/33, 60-572 Poznan, Poland.

Received: 27 March 2019 Accepted: 5 June 2019

Published online: 13 June 2019

\section{References}

1. Siegel RL, Miller KD, Jemal A. Cancer statistics, 2018. CA Cancer J Clin. 2018;68:7-30.

2. Schmoll HJ, Van Cutsem E, Stein A, Valentini V, Glimelius B, Haustermans $\mathrm{K}$, et al. ESMO Consensus Guidelines for management of patients with colon and rectal cancer. A personalized approach to clinical decision making. Ann Oncol. 2012;23:2479-516.

3. Glynne-Jones R, Wyrwicz L, Tiret E, Brown G, Rödel C, Cervantes A, et al. Rectal cancer: ESMO Clinical Practice Guidelines for diagnosis, treatment and follow-up. Ann Oncol. 2017;28:iv22-40.

4. Ogino S, Nowak JA, Hamada T, Milner DA, Nishihara R. Insights into pathogenic interactions among environment, host, and tumor at the crossroads of molecular pathology and epidemiology. Annu Rev Pathol. 2019;14:83-103.

5. Kanikarla-Marie P, Lam M, Menter DG, Kopetz S. Platelets, circulating tumor cells, and the circulome. Cancer Metastasis Rev. 2017;36:235-48.

6. Lefrançais E, Ortiz-Muñoz G, Caudrillier A, Mallavia B, Liu F, Sayah DM, et al. The lung is a site of platelet biogenesis and a reservoir for haematopoietic progenitors. Nature. 2017;544:105-9.

7. Li N. Platelets in cancer metastasis: to help the "villain" to do evil. Int J Cancer. 2016;138:2078-87.

8. Schafer Al. Thrombocytosis. N Engl J Med. 2004;350:1211-9.

9. Long Y, Wang T, Gao Q, Zhou C. Prognostic significance of pretreatment elevated platelet count in patients with colorectal cancer: a meta-analysis. Oncotarget. 2016;7:81849-61.

10. Gu D, Szallasi A. Thrombocytosis portends adverse prognosis in colorectal cancer: a meta-analysis of 5,619 patients in 16 individual studies. Anticancer Res. 2017;37:4717-26. 
11. Rao X-D, Zhang H, Xu Z-S, Cheng H, Shen W, Wang X-P. Poor prognostic role of the pretreatment platelet counts in colorectal cancer: a metaanalysis. Medicine. 2018;97:e10831.

12. Drew DA, Cao Y, Chan AT. Aspirin and colorectal cancer: the promise of precision chemoprevention. Nat Rev Cancer. 2016;16:173-86.

13. Undas A, Brummel-Ziedins KE, Mann KG. Antithrombotic properties of aspirin and resistance to aspirin: beyond strictly antiplatelet actions. Blood. 2007;109:2285-92.

14. Qiao Y, Yang T, Gan Y, Li W, Wang C, Gong Y, et al. Associations between aspirin use and the risk of cancers: a meta-analysis of observational studies. BMC Cancer. 2018;18:288.

15. Chubak J, Whitlock EP, Williams SB, Kamineni A, Burda BU, Buist DSM, et al. Aspirin for the prevention of cancer incidence and mortality: systematic evidence reviews for the U.S. Preventive Services Task Force. Ann Intern Med. 2016;164:814-25.

16. Chan AT, Ogino S, Fuchs CS. Aspirin use and survival after diagnosis of colorectal cancer. JAMA. 2009;302:649-58.

17. Liao X, Lochhead P, Nishihara R, Morikawa T, Kuchiba A, Yamauchi M, et al. Aspirin use, tumor PIK3CA mutation, and colorectal-cancer survival. N Engl J Med. 2012;367:1596-606.

18. Väyrynen JP, Kantola T, Väyrynen SA, Klintrup K, Bloigu R, Karhu T, et al. The relationships between serum cytokine levels and tumor infiltrating immune cells and their clinical significance in colorectal cancer. Int J Cancer. 2016;139:112-21.

19. Roxburgh CS, McMillan DC. The role of the in situ local inflammatory response in predicting recurrence and survival in patients with primary operable colorectal cancer. Cancer Treat Rev. 2012;38:451-66.

20. Rozek LS, Schmit SL, Greenson JK, Tomsho LP, Rennert HS, Rennert G, et al. Tumor-infiltrating lymphocytes, Crohn's-like lymphoid reaction, and survival from colorectal cancer. J Natl Cancer Inst. 2016;108:1-8.

21. McAllister SS, Weinberg RA. The tumour-induced systemic environment as a critical regulator of cancer progression and metastasis. Nat Cell Biol. 2014;16:717-27

22. Kantola T, Klintrup K, Väyrynen JP, Vornanen J, Bloigu R, Karhu T, et al. Stage-dependent alterations of the serum cytokine pattern in colorectal carcinoma. Br J Cancer. 2012;107:1729-36.

23. Väyrynen JP, Tuomisto A, Väyrynen SA, Klintrup K, Karhu T, Mäkelä J, et al. Preoperative anemia in colorectal cancer: relationships with tumor characteristics, systemic inflammation, and survival. Sci Rep. 2018;8:1126.

24. Sirniö P, Tuomisto A, Tervahartiala T, Sorsa T, Klintrup K, Karhu T, et al. Highserum MMP-8 levels are associated with decreased survival and systemic inflammation in colorectal cancer. Br J Cancer. 2018;119:213-9.

25. Sirniö P, Väyrynen JP, Klintrup K, Mäkelä J, Mäkinen MJ, Karttunen TJ, et al. Decreased serum apolipoprotein A1 levels are associated with poor survival and systemic inflammatory response in colorectal cancer. Sci Rep. 2017:7:5374.

26. Väyrynen JP, Mutt SJ, Herzig K-H, Väyrynen SA, Kantola T, Karhu T, et al. Decreased preoperative serum 25-Hydroxyvitamin D levels in colorectal cancer are associated with systemic inflammation and serrated morphology. Sci Rep. 2016;6:36519.

27. McShane LM, Altman DG, Sauerbrei W, Taube SE, Gion M, Clark GM. REporting recommendations for tumour MARKer prognostic studies (REMARK). Br J Cancer. 2005;93:387-91.

28. Väyrynen JP, Vornanen J, Tervahartiala T, Sorsa T, Bloigu R, Salo T, et al. Serum MMP-8 levels increase in colorectal cancer and correlate with disease course and inflammatory properties of primary tumors. Int J Cancer. 2012;131:E463-74.

29. McMillan DC. The systemic inflammation-based Glasgow Prognostic Score: a decade of experience in patients with cancer. Cancer Treat Rev. 2013:39:534-40.

30. Sirniö P, Väyrynen JP, Klintrup K, Mäkelä J, Karhu T, Herzig K-H, et al. Alterations in serum amino-acid profile in the progression of colorectal cancer: associations with systemic inflammation, tumour stage and patient survival. Br J Cancer. 2019:120:238-46.

31. Väyrynen SA, Väyrynen JP, Klintrup K, Mäkelä J, Karttunen TJ, Tuomisto A, et al. Clinical impact and network of determinants of tumour necrosis in colorectal cancer. Br J Cancer. 2016;114:1334-42.

32. Väyrynen JP, Sajanti SA, Klintrup K, Mäkelä J, Herzig K-H, Karttunen TJ, et al. Characteristics and significance of colorectal cancer associated lymphoid reaction. Int J Cancer. 2014;134:2126-35.
33. Sajanti SA, Väyrynen JP, Sirniö P, Klintrup K, Mäkelä J, Tuomisto A, et al. Annexin A10 is a marker for the serrated pathway of colorectal carcinoma. Virchows Arch. 2015;466:5-12.

34. Sajanti SA, Sirniö P, Väyrynen JP, Tuomisto A, Klintrup K, Mäkelä J, et al. VE1 immunohistochemistry accurately detects BRAF V600E mutations in colorectal carcinoma and can be utilized in the detection of poorly differentiated colorectal serrated adenocarcinoma. Virchows Arch. 2014;464:637-43

35. Väyrynen JP, Vornanen JO, Sajanti S, Böhm JP, Tuomisto A, Mäkinen MJ. An improved image analysis method for cell counting lends credibility to the prognostic significance of T cells in colorectal cancer. Virchows Arch. 2012;460:455-65.

36. Väyrynen JP, Tuomisto A, Klintrup K, Mäkelä J, Karttunen TJ, Mäkinen MJ. Detailed analysis of inflammatory cell infiltration in colorectal cancer. $\mathrm{Br}$ J Cancer. 2013;109:1839-47.

37. Shannon P, Markiel A, Ozier O, Baliga NS, Wang JT, Ramage D, et al. Cytoscape: a software environment for integrated models of biomolecular interaction networks. Genome Res. 2003;13:2498-504.

38. Benjamin DJ, Berger JO, Johannesson M, Nosek BA, Wagenmakers E-J, Berk R, et al. Redefine statistical significance. Nat Hum Behav. 2018;2:6-10.

39. Petrelli F, Barni S, Coinu A, Bertocchi P, Borgonovo K, Cabiddu M, et al. The modified glasgow prognostic score and survival in colorectal cancer: a pooled analysis of the literature. Rev Recent Clin Trials. 2015;10:135-41.

40. Thorpe S, Fize D, Marlot C. Speed of processing in the human visual system. Nature. 1996;381:520-2.

41. Blair P, Flaumenhaft R. Platelet alpha-granules: basic biology and clinical correlates. Blood Rev. 2009:23:177-89.

42. Damås JK, Waehre T, Yndestad A, Otterdal K, Hognestad A, Solum NO, et al. Interleukin-7-mediated inflammation in unstable angina: possible role of chemokines and platelets. Circulation. 2003;107:2670-6.

43. Biancotto A, Feng X, Langweiler M, Young NS, McCoy JP. Effect of anticoagulants on multiplexed measurement of cytokine/chemokines in healthy subjects. Cytokine. 2012;60:438-46.

44. Lin J, Zhu Z, Xiao H, Wakefield MR, Ding VA, Bai Q, et al. The role of IL-7 in immunity and cancer. Anticancer Res. 2017:37:963-7.

45. Heldin $\mathrm{C}-\mathrm{H}$. Targeting the PDGF signaling pathway in tumor treatment. Cell Commun Signal. 2013;11:97.

46. Francipane MG, Alea MP, Lombardo Y, Todaro M, Medema JP, Stassi G. Crucial role of interleukin-4 in the survival of colon cancer stem cells. Cancer Res. 2008;68:4022-5.

47. Bester J, Pretorius E. Effects of IL-1 $\beta, I L-6$ and IL-8 on erythrocytes, platelets and clot viscoelasticity. Sci Rep. 2016;6:32188.

48. Väyrynen JP, Tuomisto A, Mäkinen MJ. Regulatory mechanisms of T cell activation-from basic research discoveries to a new principle of cancer therapy and the Nobel Prize. Acta Physiol. 2019;225:e13224.

49. Maby P, Tougeron D, Hamieh M, Mlecnik B, Kora H, Bindea G, et al. Correlation between density of CD8+ T-cell infiltrate in microsatellite unstable colorectal cancers and frameshift mutations: a rationale for personalized immunotherapy. Cancer Res. 2015;75:3446-55.

50. Wirta E-V, Seppälä T, Friman M, Väyrynen J, Ahtiainen M, Kautiainen H, et al. Immunoscore in mismatch repair-proficient and -deficient colon cancer. J Pathol Clin Res. 2017;3:203-13.

51. Bleeker JS, Hogan WJ. Thrombocytosis: diagnostic evaluation, thrombotic risk stratification, and risk-based management strategies. Thrombosis. 2011;2011:536062.

52. Moreno Chulilla JA, Romero Colás MS, Martín MG. Classification of anemia for gastroenterologists. World J Gastroenterol. 2009;15:4627-37.

53. McSorley ST, Johnstone M, Steele CW, Roxburgh CSD, Horgan PG, McMillan DC, et al. Normocytic anaemia is associated with systemic inflammation and poorer survival in patients with colorectal cancer treated with curative intent. Int J Colorectal Dis. 2019;34:401-8.

54. Wilson MJ, van Haaren M, Harlaar JJ, Park HC, Bonjer HJ, Jeekel J, et al. Long-term prognostic value of preoperative anemia in patients with colorectal cancer: a systematic review and meta-analysis. Surg Oncol. 2017;26:96-104.

55. Tokunaga R, Nakagawa S, Miyamoto Y, Ohuchi M, Izumi D, Kosumi K, et al. The impact of preoperative anaemia and anaemic subtype on patient outcome in colorectal cancer. Colorectal Dis. 2019;21:100-9.

56. Gargiulo G, Windecker S, Vranckx P, Gibson CM, Mehran R, Valgimigli M. A critical appraisal of aspirin in secondary prevention: is less more? Circulation. 2016:134:1881-906. 
57. Camp RL, Neumeister V, Rimm DL. A decade of tissue microarrays: progress in the discovery and validation of cancer biomarkers. J Clin Oncol. 2008;26:5630-7.

\section{Publisher's Note}

Springer Nature remains neutral with regard to jurisdictional claims in published maps and institutional affiliations.
Ready to submit your research? Choose BMC and benefit from:

- fast, convenient online submission

- thorough peer review by experienced researchers in your field

- rapid publication on acceptance

- support for research data, including large and complex data types

- gold Open Access which fosters wider collaboration and increased citations

- maximum visibility for your research: over $100 \mathrm{M}$ website views per year

At BMC, research is always in progress.

Learn more biomedcentral.com/submissions 